\title{
Colon capsule endoscopy: toward the future
}

\author{
Naoki Muguruma ${ }^{1}$ (D) $\cdot$ Kumiko Tanaka $^{1} \cdot$ Satoshi Teramae $^{1} \cdot$ Tetsuji Takayama $^{1}$
}

Received: 19 December 2016/Accepted: 20 December 2016/Published online: 13 January 2017

(C) Japanese Society of Gastroenterology 2017

\begin{abstract}
Colon capsule endoscopy is a wireless and minimally invasive technique for visualization of the whole colon. With recent improvements of technical features in second-generation systems, a more important role for colon capsule endoscopy is rapidly emerging. Although several limitations and drawbacks are yet to be resolved, its usefulness as a tool for colorectal cancer screening and monitoring disease activity in inflammatory bowel diseases has become more apparent with increased use. Further investigations, including multicenter trials, are required to evaluate the substantial role of the colon capsule in managing colorectal diseases.
\end{abstract}

Keywords Capsule endoscopy $\cdot$ Colonoscopy $\cdot$ Colorectal cancer $\cdot$ Polyp $\cdot$ IBD

\section{Introduction}

Colorectal cancer (CRC) is one of the leading causes of cancer-related death in both men and women in Western [1] and Asian countries, including Japan [2, 3]. In Japan, CRC screening programs organized by the national government for colorectal cancer based on fecal occult blood testing (FOBT) started in 1992, and the incidence of CRC halted its increase shortly thereafter. Japanese population-based CRC screening is offered to those aged 40 years and over, and total colonoscopy is performed for those with positive fecal

Naoki Muguruma

muguruma.clin.med@gmail.com

1 Department of Gastroenterology and Oncology, Tokushima University Graduate School of Biomedical Sciences, 3-18-15 Kuramoto-cho, Tokushima 770-8503, Japan immunochemical test (FIT) result [4]. Although use of FOBT or FIT has been demonstrated to reduce mortality from CRC in randomized controlled trials [5], the specificity and sensitivity of fecal occult blood screening are substantially limited. Colonoscopy is the gold-standard modality for early detection of adenoma, the colorectal premalignant lesion, thereby leading to prevention of CRC [6]. However, the acceptance rate for CRC screening is still low in most Western countries [7] as well as Japan. This is probably due to the invasive nature of colonoscopy, unpleasant perceptions, and complications such as bleeding or perforation [8].

Colon capsule endoscopy (CCE) was introduced in 2006 as a minimally invasive and wireless technique for imaging the colon [9]. To increase the accuracy of CCE, secondgeneration CCE was developed and eventually introduced in 2009. This modality is a novel and promising technology that can be useful for screening and monitoring of colorectal diseases [10]. In this review article, we comprehensively analyze recent reports and discuss the potential roles and future perspectives of CCE.

\section{Technical features}

The PillCam colon capsule endoscope (Covidien-Medtronic, Dublin, Ireland) is now available in its secondgeneration form (CCE-2). There are three main components to this system: (1) an ingestible capsule endoscope $\left(11.6 \times 31.5 \mathrm{~mm}^{2}\right.$ in size, equipped with two head cameras with $172^{\circ}$ angle of view), (2) a sensing system with sensing pads or a sensing belt to attach to the patient, a data recorder, and a battery pack, and (3) a personal computer workstation with software (RAPID 8; Fig. 1a-c). Compared with the first-generation CCE with $156^{\circ}$ angle view, the new model allows for almost $360^{\circ}$ visual coverage of 


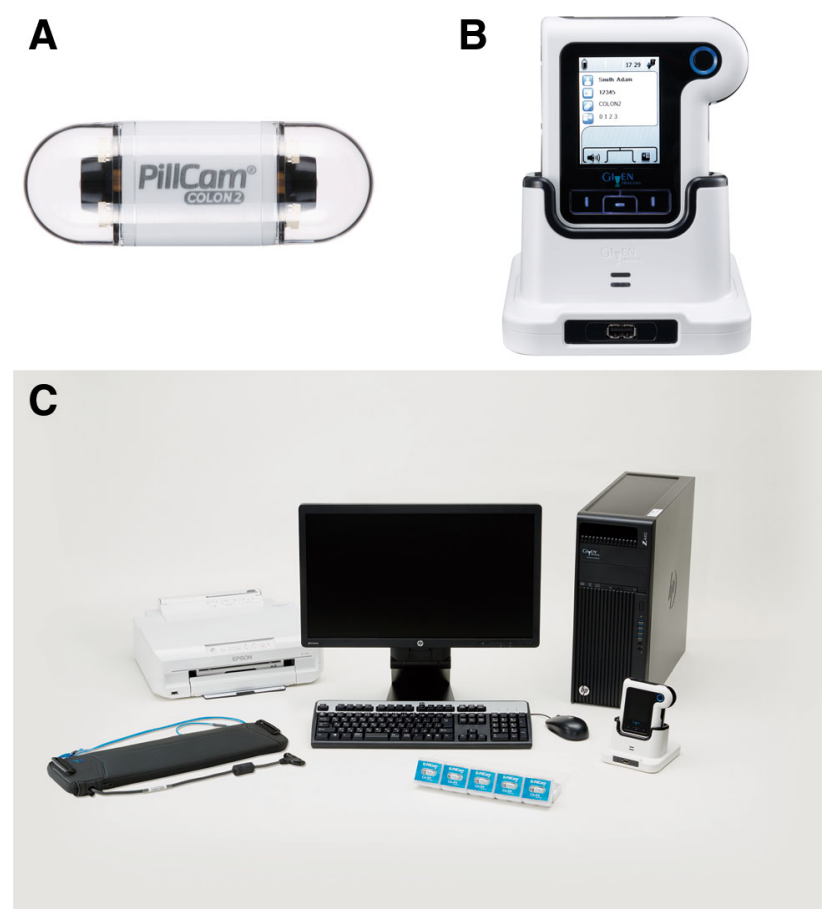

Fig. 1 Second-generation colon capsule endoscopy system: a capsule with two cameras incorporated, $\mathbf{b}$ data recorder with real-time viewer, and $\mathbf{c}$ workstation (RAPID 8)

the colon. The data recorder is incorporated within handheld viewers that allow real-time review of images during examinations. It also has the unique feature of an adaptive frame rate (AFR). The AFR function is activated using a bidirectional communication system between the capsule and data recorder. It automatically determines the frame rate, which ranges from 4 per second when stable to 35 per second while in rapid motion. This function not only improves battery life, but also reduces "skipped" visualization of the colon. The resolution of the CCE- 2 image is below $0.1 \mathrm{~mm}$, with magnification range of about $1-8$ (Fig. 2a). The latest RAPID software includes a graphical interface tool for polyp size estimation (Fig. 2b), as well as image enhancement, including flexible spectral imaging color enhancement (FICE; Fig. 2c, d). Moreover, the capsule is capable of giving feedback to the patient regarding its location via vibrations, and displays instructions when small bowel mucosa is detected [11].

\section{Indications and contraindications}

Current indications for CCE in Japan are (1) incomplete colonoscopy due to technical difficulties such as scope looping, and (2) presumed difficulty in conventional colonoscopy because of colonic adhesion due to previous abdominal surgery. Contraindications for CCE are comparable to those for small bowel capsule endoscopy [12].
However, CCE examinations should not be performed in patients (1) with known or suspected gastrointestinal obstruction, stricture, or fistula, because of the risk of CCE retention, (2) with cardiac pacemakers or other implanted electromedical devices because of the microwaves transmitted by the CCE, (3) with swallowing disorders because of risk of aspiration, and (4) who are pregnant $[13,14]$.

\section{Preparation}

Accurate diagnosis can only be achieved when the colon is completely free of stool during the colonic examination. Unlike conventional colonoscopy, adequate bowel preparation is crucial to achieve successful CCE because additional cleaning maneuvers, such as washing and suctioning during the procedure, are not available [13]. In addition, the capsule transit time, which allows its excretion from the rectum within the capsule's battery life, is also important $[15,16]$. There is an apparently positive correlation between examination sensitivity for colonic lesions and the degree of cleansing of the colon [17]. Subjects are encouraged to start a low-residue diet 2 days before CCE and a clear liquid diet the day before CCE. There are various diet protocols in place for CCE, depending on the institution, mainly consisting of polyethylene glycol (PEG) solution and boosters with sodium phosphate [17, 18]. Sodium phosphate can accelerate the bowel transit time of the capsule but may cause serious complications such as acute renal failure and electrolyte imbalance. Patients who are at risk of sodium phosphate toxicity need to undergo alternative booster preparations such as magnesium citrate [19-21]. Gastrografin, which is a contrast medium for the digestive tract, is an alternative to sodium phosphate in the bowel preparation regimen for CCE [22]. Additional agents may also be used: emptying prokinetics for gastric motility and a bisacodyl suppository [23]. Aromatic castor oil, a vegetable oil obtained by pressing the seeds of the plant, may also act as a booster (personal communication). Patient tolerance of the bowel preparation used is a substantial concern, because reluctance to repeat such intensive preparation has been widely reported [24]. Unfortunately, the optimal preparation method that is both effective in cleansing for successful CCE and tolerable by patients has yet to be determined.

\section{CCE for detection of colon polyps}

Since the introduction of the CCE-2 with improved performance as described above, the accuracy of CCE for polyp detection has increased [10, 13, 25, 26]. To date, five studies have been published on polyp detection by CCE- 2 
Fig. 2 a High-resolution image of a colon polyp captured by CCE-2. b Polyp size estimated with graphics tool software. c Image of angioectasia detected under white light. d Enhanced image using flexible spectral imaging color enhancement (FICE)
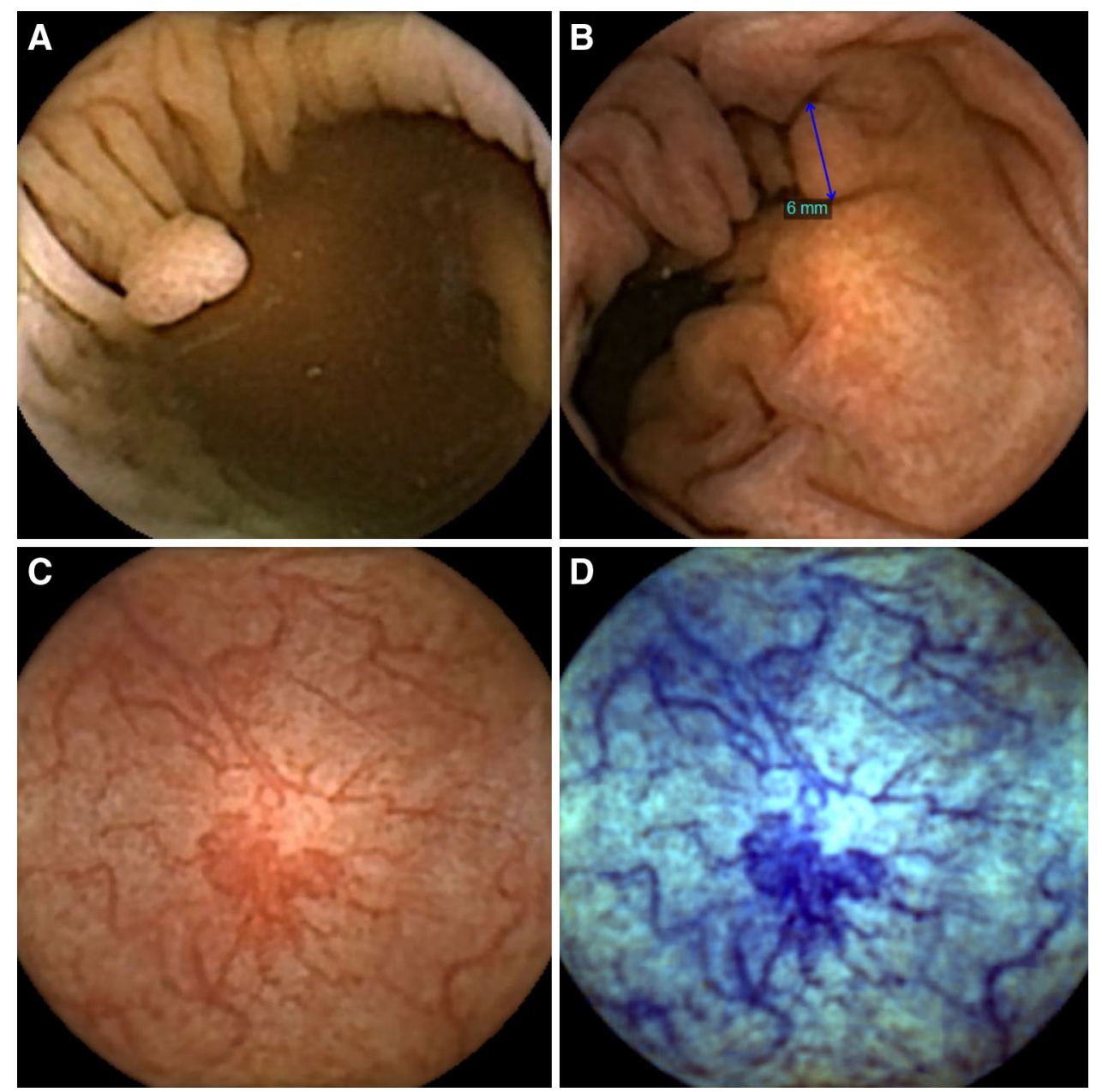

Table 1 Diagnostic accuracy of CCE-2 versus colonoscopy for detecting colon polyps

\begin{tabular}{llllllll}
\hline Author [Ref.] & Year & No. & Completion (\%) & $\geq 6 \mathrm{~mm}$ & & $\geq 10 \mathrm{~mm}$ \\
\cline { 6 - 8 } & & & & Sensitivity (\%) & Specificity (\%) & Sensitivity (\%) & Specificity (\%) \\
\hline Eliakim [27] & 2009 & 98 & 81 & 89 & 76 & 88 & 89 \\
Spada [28] & 2011 & 109 & 81 & 84 & 64 & 88 & 95 \\
Rex [31] & 2015 & 695 & 91 & 88 & 82 & 92 & 95 \\
\hline
\end{tabular}

compared with conventional colonoscopy [27-31]. These studies highlighted the considerably high sensitivity and specificity of CCE-2. Three landmark studies that recruited more than 100 patients reported sensitivity and specificity ranging from 84 to 89 and 64 to $95 \%$, respectively (Table 1 ). The relatively low specificity was mainly due to size discrepancy rather than false positives. However, there were a few adverse effects, usually related to bowel preparation. A metaanalysis of over 2400 subjects revealed sensitivity for $6 \mathrm{~mm}$ and larger polyps of $58 \%$ for the firstgeneration $\mathrm{CE}$ and $86 \%$ for the CCE-2 [32]. Based on these results, combined with its safety profile, it was concluded that the colon capsule may be a suitable alternative to colonoscopy for colon polyps, particularly in patients unwilling to undergo colonoscopy or for those in whom it is technically not feasible.

\section{CCE in IBD}

Recently, several studies on surveillance of inflammatory bowel diseases (IBD) have been published, mainly on ulcerative colitis (UC) [33-37]. However, data regarding use of CCE in patients with IBD are insufficient because histological verification is required for diagnosis of IBD. A possible role for CCE in the field of IBD is for evaluation 
of mucosal healing while monitoring disease activity [13]. In a large study conducted by Sung and colleagues using first-generation CCE [33], the sensitivity and specificity of CCE for detection of active ulcerative colitis was 89 and $75 \%$, respectively, when compared with conventional colonoscopy. In a recent study of pediatric patients using CCE-2 [36], the sensitivity and specificity for disease activity were 96 and $100 \%$, respectively. However, whether CCE is superior to conventional colonoscopy for assessment of disease activity and extent in UC remains controversial [34].

Although literature on use of CCE in Crohn's disease (CD) is sparse due to the concern of capsule retention caused by strictures, more recent studies demonstrate the safety and feasibility of use of CE in this disease [38-42]. In a multicenter pilot study, CCE-2 detected colonic ulcerations with $86 \%$ sensitivity and $40 \%$ specificity, and no adverse events were observed. As capsule use in evaluating the small bowel of patients with $\mathrm{CD}$ has become well established, once a small bowel stenosis is reliably excluded by preceding patency capsule ingestion, CCE can be used as a panenteroscopic test in this disease [43].

\section{Complications}

CCE has been shown to be a safe procedure so far, with complications mostly due to bowel cleansing. In an analysis of over 1600 reported cases involving both first- and second-generation CEs, the minor complication rate was $4.1 \%$, while the major complication rate was $0.49 \%$, not being directly related to CCE [25]. Reports stated that the major complication of perforation was derived from the particular performance of the colonoscopy, including the therapeutic interventions undertaken [44-46] (Table 2). Some case reports raised the possibility of capsule aspiration [47] and retention in a diverticulum [48]. In any case, endoscopic or surgical interventions may be necessary to address any complications such as retention or aspiration. After ingesting the CCE and until it is successfully excreted, the patient should not approach any source of powerful electromagnetic fields such as magnetic resonance imaging scanners, as instructed by the provider.

\section{Future perspectives}

CCE examination has been estimated to cost approximately $\$ 950$ in the USA, $€ 700$ in Europe, and $¥ 120,000$ (without medical insurance) in Japan. Cost-performance issues underlie when this novel technology will be introduced into CRC screening [49]. For polyp detection, CT colonography (CTC) has also been recommended as an imaging modality of choice in the case of incomplete colonoscopy in the USA [50]. One recent clinical trial demonstrated that both CCE- 2 and CTC detected polyps $6 \mathrm{~mm}$ and larger with high levels of accuracy; CCE-2 seemed to be better tolerated than CTC [51]. Another prospective study for patients with incomplete colonoscopy demonstrated that the diagnostic yield of CCE was superior to that of CTC [23]. However, the advantages and disadvantages of CCE should be discussed compared with those of CTC, which seems to be a cost-effective option in CRC screening.

During conventional colonoscopy, the colon is distended by air or carbon dioxide, but during CCE, it is naturally distended by water, ensuring lesions are not so stretched in the colonic wall [52]. Flat or sessile lesions require special attention by endoscopists because they have higher risk of cancer than polypoid lesions. It is often difficult to detect sessile serrated adenoma/polyps (SSA/Ps) with conventional colonoscopy because the form of the lesion is typically flat and the color is similar to the background mucosa or more faded [53]. Image enhancement techniques have been reported to be useful in detecting serrated lesions [54]. Importantly, prospective trials evaluating the accuracy of CCE-2, with or without spectral image color enhancement, in the detection of flat and sessile lesions are greatly needed.

Training systems for CCE also need to be developed. Several training programs have been established for small bowel CE, but the method of reading and interpretation of

Table 2 Complication rates from reported studies involving over 100 cases

\begin{tabular}{llllll}
\hline Author [Ref.] & Year & No. & \multicolumn{2}{l}{ Complication rate } & Cause of major complication \\
\cline { 5 - 5 } & & & Major $(\%)$ & Minor (\%) & \\
\hline Van Gossum [17] & 2009 & 320 & 0 & 2.9 & \\
Eliakim [27] & 2009 & 104 & 0.96 & 7.7 & Urinary retention \\
Gay [44] & 2010 & 128 & 0 & 0 & \\
Sacher-Huvelin [45] & 2010 & 545 & 0.5 & 3.5 & Cardiac failure, colonic perforation ${ }^{\text {a }}$, bleeding after mucosectomy \\
Spada [28] & 2011 & 109 & 0.85 & 6.8 & Perforation after colon polypectomy \\
Herrerías-Gutiérrez [46] & 2011 & 144 & 0 & 0 &
\end{tabular}

${ }^{a}$ Potentially related to bowel preparation 
results in CCE is totally different from that in small bowel CE because of its characteristic functions, double cameras, and AFR. A pilot study using an electric learning system for CCE (ELCCE) has been reported to be effective for improving reading competence for CCE. Hands-on training for trainees also seems useful to shorten the learning curve in achieving CE competency [55-57]. Apart from this, with $\mathrm{CCE}$, the entire gastrointestinal tract is visualized with potential extracolonic findings and pathology. This may be beneficial for patients receiving $\mathrm{CCE}$ as a panendoluminal examination but impose an excessive burden on interpreters.

The human colon is a relatively large-sized organ whose complete visualization using a passive capsule is quite difficult. A capsule maneuvered with an external magnetic field, a so-called magnetically controlled capsule (MCC), has recently been developed, and several trials have reported promising results, mainly for gastric lesions [58]. This novel MCC has shown high diagnostic accuracy compared with conventional gastroscopy and has a very low complication rate. In the colon, the maneuverability and safety of MCC have also been demonstrated [59], and increased accuracy in detecting and monitoring colorectal lesions can be expected in the near future.

\section{Conclusions}

Although several issues such as cost-performance, practical implementation, training programs, and patient preference remain to be resolved, the technical performance of second-generation CCE has markedly improved. Given the expectation of further evolution in medical device technology, CCE appears to be a novel, promising technique that is noninvasive and painless and therefore suitable for evaluation of colorectal diseases. Further investigations, such as prospective randomized trials with a large number of subjects, are required to establish the firm inclusion of $\mathrm{CCE}$ in diagnostic algorithms for colorectal diseases.

\section{Compliance with ethical standards}

Conflict of interest: All authors declare that they have no conflict of interest

Human Rights: All procedures followed have been performed in accordance with the ethical standards laid down in the 1964 Declaration of Helsinki and its later amendments.

Informed Consent: No informed consent.

\section{References}

1. Siegel R, DeSantis C, Jemal A. Colorectal cancer statistics, 2014. CA Cancer J Clin. 2014;64:104-17.
2. Sung JJY, Lau JYW, Young GP, et al. Asia Pacific consensus recommendations for colorectal cancer screening. Gut. 2008;57:1166-76.

3. Katanoda K, Hori M, Matsuda T, et al. An updated report on the trends in cancer incidence and mortality in Japan, 1958-2013. Jpn J Clin Oncol. 2015;45:390-401.

4. Sekiguchi M, Igarashi A, Matsuda T, et al. Optimal use of colonoscopy and fecal immunochemical test for population-based colorectal cancer screening: a cost-effectiveness analysis using Japanese data. Jpn J Clin Oncol. 2016;46:116-25.

5. Lee KJ, Inoue M, Otani T, et al. Colorectal cancer screening using fecal occult blood test and subsequent risk of colorectal cancer: a prospective cohort study in Japan. Cancer Detect Prev. 2007;31:3-11.

6. Edwards BK, Ward E, Kohler BA, et al. Annual report to the nation on the status of cancer, 1975-2006, featuring colorectal cancer trends and impact of interventions (risk factors, screening, and treatment) to reduce future rates. Cancer. 2010;116:544-73.

7. Bujanda L, Sarasqueta C, Zubiaurre L, et al. Low adherence to colonoscopy in the screening of first-degree relatives of patients with colorectal cancer. Gut. 2007;56:1714-8.

8. Kim DH, Pickhardt PJ, Taylor AJ, et al. CT colonography versus colonoscopy for the detection of advanced neoplasia. N Engl J Med. 2007;357:1403-12.

9. Schoofs N, Devière J, Van Gossum A. PillCam colon capsule endoscopy compared with colonoscopy for colorectal tumor diagnosis: a prospective pilot study. Endoscopy. 2006;38:971-7.

10. Carter D, Eliakim R. PillCam colon capsule endoscopy (PCCE) in colonic diseases. Ann Transl Med. 2016;4:307.

11. Spada C, Hassan C, Costamagna G. Colon capsule endoscopy. Gastrointest Endosc Clin N Am. 2015;25:387-401.

12. Ladas SD, Triantafyllou K, Spada C, et al. European Society of Gastrointestinal Endoscopy (ESGE): recommendations (2009) on clinical use of video capsule endoscopy to investigate smallbowel, esophageal and colonic diseases. Endoscopy. 2010;42:220-7.

13. Han YM, Im JP. Colon capsule endoscopy: where are we and where are we going. Clin Endosc. 2016;49:449-53.

14. Wang A, Banerjee S, Barth BA, et al. Wireless capsule endoscopy. Gastrointest Endosc. 2013;78:805-15.

15. Spada C, Riccioni ME, Hassan C, et al. PillCam colon capsule endoscopy: a prospective, randomized trial comparing two regimens of preparation. J Clin Gastroenterol. 2011;45:119-24.

16. Spada $C$, Hassan $C$, Ingrosso $M$, et al. A new regimen of bowel preparation for PillCam colon capsule endoscopy: a pilot study. Dig Liver Dis. 2011;43:300-4.

17. Van Gossum A, Munoz-Navas M, Fernandez-Urien I, et al. Capsule endoscopy versus colonoscopy for the detection of polyps and cancer. N Engl J Med. 2009;361:264-70.

18. Sieg A, Friedrich K, Sieg U. Is PillCam COLON capsule endoscopy ready for colorectal cancer screening? A prospective feasibility study in a community gastroenterology practice. Am J Gastroenterol. 2009;104:848-54.

19. Spada C, Hassan C, Galmiche JP, et al. Colon capsule endoscopy: European Society of Gastrointestinal Endoscopy (ESGE) guideline. Endoscopy. 2012;44:527-36.

20. Hassan C, Bretthauer M, Kaminski MF, et al. Bowel preparation for colonoscopy: European Society of Gastrointestinal Endoscopy (ESGE) guideline. Endoscopy. 2013;45:142-50.

21. Mathus-Vliegen E, Pellisé M, Heresbach D, et al. Consensus guidelines for the use of bowel preparation prior to colonic diagnostic procedures: colonoscopy and small bowel video capsule endoscopy. Curr Med Res Opin. 2013;29:931-45.

22. Togashi K, Fujita T, Utano K, et al. Gastrografin as an alternative booster to sodium phosphate in colon capsule endoscopy: safety and efficacy pilot study. Endosc Int Open. 2015;3:E659-61. 
23. Spada C, Hassan C, Barbaro B, et al. Colon capsule versus CT colonography in patients with incomplete colonoscopy: a prospective, comparative trial. Gut. 2015;64:272-81.

24. Adrián-de-Ganzo Z, Alarcón-Fernández $\mathrm{O}$, Ramos L, et al. Uptake of colon capsule endoscopy vs colonoscopy for screening relatives of patients with colorectal cancer. Clin Gastroenterol Hepatol. 2015;13:2293-301.e1.

25. Tal AO, Vermehren J, Albert JG. Colon capsule endoscopy: current status and future directions. World J Gastroenterol. 2014;20:16596-602.

26. Friedel D, Modayil R, Stavropoulos S. Colon capsule endoscopy: review and perspectives. Gastroenterol Res Pract. 2016;2016:9643162.

27. Eliakim R, Yassin K, Niv Y, et al. Prospective multicenter performance evaluation of the second-generation colon capsule compared with colonoscopy. Endoscopy. 2009;41:1026-31.

28. Spada C, Hassan C, Munoz-Navas M, et al. Second-generation colon capsule endoscopy compared with colonoscopy. Gastrointest Endosc. 2011;74:581-9.e1.

29. Holleran G, Leen R, O'Morain C, et al. Colon capsule endoscopy as possible filter test for colonoscopy selection in a screening population with positive fecal immunology. Endoscopy. 2014;46:473-8.

30. Hagel AF, Gäbele E, Raithel M, et al. Colon capsule endoscopy: detection of colonic polyps compared with conventional colonoscopy and visualization of extracolonic pathologies. Can J Gastroenterol Hepatol. 2014;28:77-82.

31. Rex DK, Adler SN, Aisenberg J, et al. Accuracy of capsule colonoscopy in detecting colorectal polyps in a screening population. Gastroenterology. 2015;148:948-57.e2.

32. Spada C, Pasha SF, Gross SA, et al. Accuracy of first- and second-generation colon capsules in endoscopic detection of colorectal polyps: a systematic review and meta-analysis. Clin Gastroenterol Hepatol. 2016;14:1533-43.e8.

33. Sung J, Ho KY, Chiu HM, et al. The use of Pillcam colon in assessing mucosal inflammation in ulcerative colitis: a multicenter study. Endoscopy. 2012;44:754-8.

34. Meister T, Heinzow HS, Domagk D, et al. Colon capsule endoscopy versus standard colonoscopy in assessing disease activity of ulcerative colitis: a prospective trial. Tech Coloproctol. 2013; 17:641-6.

35. Ye CA, Gao YJ, Ge ZZ, et al. PillCam colon capsule endoscopy versus conventional colonoscopy for the detection of severity and extent of ulcerative colitis. J Dig Dis. 2013;14:117-24.

36. Oliva S, Di Nardo G, Hassan C, et al. Second-generation colon capsule endoscopy vs. colonoscopy in pediatric ulcerative colitis: a pilot study. Endoscopy. 2014;46:485-92.

37. Hosoe N, Matsuoka K, Naganuma M, et al. Applicability of secondgeneration colon capsule endoscope to ulcerative colitis:a clinical feasibility study. J Gastroenterol Hepatol. 2013;28:1174-9.

38. D'Haens G, Löwenberg M, Samaan MA, et al. Safety and feasibility of using the second-generation Pillcam colon capsule to assess active colonic Crohn's disease. Clin Gastroenterol Hepatol. 2015;13:1480-6.e3.

39. Boal Carvalho P, Rosa B, Dias de Castro F, et al. PillCam COLON2 in Crohn's disease: a new concept of panenteric mucosal healing assessment. World J Gastroenterol. 2015;21:7233-41.

40. Niv Y, Gal E, Gabovitz V, et al (2016) Capsule endoscopy Crohn's disease activity Index (CECDAIic or Niv Score) for the small bowel and colon. J Clin Gastroenterol. doi:10.1097/MCG. 0000000000000720

41. Oliva S, Cucchiara S, Civitelli F, et al. Colon capsule endoscopy compared with other modalities in the evaluation of pediatric Crohn's disease of the small bowel and colon. Gastrointest Endosc. 2016;83:975-83.

42. Negreanu L, Smarandache G, Mateescu RB. Role of capsule endoscopy PillcamCOLON 2 in patients with known or suspected
Crohn's disease who refused colonoscopy or underwent incomplete colonoscopic exam: a case series. Tech Coloproctol. 2014; 18:277-83.

43. Hall B, Holleran G, McNamara D. PillCam COLON 2(C) as a pan-enteroscopic test in Crohn's disease. World J Gastrointest Endosc. 2015;7:1230-2.

44. Gay G, Delvaux M, Frederic M, et al. Could the colonic capsule PillCam Colon be clinically useful for selecting patients who deserve a complete colonoscopy?: results of clinical comparison with colonoscopy in the perspective of colorectal cancer screening. Am J Gastroenterol. 2010;105:1076-86.

45. Sacher-Huvelin S, Coron E, Gaudric M, et al. Colon capsule endoscopy vs. colonoscopy in patients at average or increased risk of colorectal cancer. Aliment Pharmacol Ther. 2010;32:1145-53.

46. Herrerías-Gutiérrez JM, Argüelles-Arias F, Caunedo-Álvarez A, et al. PillCam colon capsule for the study of colonic pathology in clinical practice. Study of agreement with colonoscopy. Rev Esp Enferm Dig. 2011;103:69-75.

47. Amarna M, Vanlandingham A, Brahmbhatt $P$, et al. Late presentation of capsule endoscope aspiration with successful extraction by flexible bronchoscopy utilizing a snare wire loop. Endoscopy. 2015;47(Suppl 1 UCTN):E6-7.

48. Courcoutsakis N, Pitiakoudis M, Mimidis K, et al. Capsule retention in a giant Meckel's diverticulum containing multiple enteroliths. Endoscopy. 2011;43(Suppl 2 UCTN):E308-9.

49. Hassan C, Zullo A, Winn S, et al. Cost-effectiveness of capsule endoscopy in screening for colorectal cancer. Endoscopy. 2008;40:414-21.

50. AGA Clinical Practice and Economics Committee. Position of the American Gastroenterological Association (AGA) Institute on computed tomographic colonography. Gastroenterology. 2006;131:1627-8.

51. Rondonotti E, Borghi C, Mandelli G, et al. Accuracy of capsule colonoscopy and computed tomographic colonography in individuals with positive results from the fecal occult blood test. Clin Gastroenterol Hepatol. 2014;12:1303-10.

52. Spada C, Barbaro F, Andrisani G, et al. Colon capsule endoscopy: what we know and what we would like to know. World J Gastroenterol. 2014;20:16948-55.

53. Okamoto K, Kitamura S, Kimura T, et al. Clinicopathological characteristics of serrated polyps as precursors to colorectal cancer: current status and management. J Gastroenterol Hepatol. 2016. doi:10.1111/jgh.13482.

54. Hazewinkel Y, Tytgat KM, van Leerdam ME, et al. Narrow-band imaging for the detection of polyps in patients with serrated polyposis syndrome: a multicenter, randomized, back-to-back trial. Gastrointest Endosc. 2015;81:531-8.

55. Lim YJ, Joo YS, Jung DY, et al. Learning curve of capsule endoscopy. Clin Endosc. 2013;46:633-6.

56. Albert JG, Humbla O, McAlindon ME, et al. A simple evaluation tool (ET-CET) indicates increase of diagnostic skills from small bowel capsule endoscopy training courses: a prospective observational European multicenter study. Medicine (Baltimore). 2015;94:e1941.

57. Watabe H, Nakamura T, Yamada A, et al. Assessment of an electronic learning system for colon capsule endoscopy: a pilot study. J Gastroenterol. 2016;51:579-85.

58. Liao Z, Hou X, Lin-Hu EQ, et al. Accuracy of magnetically controlled capsule endoscopy, compared with conventional gastroscopy, in detection of gastric diseases. Clin Gastroenterol Hepatol. 2016;14:1266-73.e1.

59. Gu H, Zheng H, Cui X, Huang Y, Jiang B (2016) Maneuverability and safety of a magnetic-controlled capsule endoscopy system to examine the human colon under real-time monitoring by colonoscopy: a pilot study (with video). Gastrointest Endosc. doi:10.1016/j.gie.2016.07.053. 